\title{
Two-Photon Imaging within the Murine Thorax without Respiratory and Cardiac Motion Artifact
}

\author{
Robert G. Presson Jr, ${ }^{* \dagger}$ Mary Beth Brown, ${ }^{* \neq}$ \\ Amanda J. Fisher, ${ }^{* \dagger}$ Ruben M. Sandoval, ${ }^{\ddagger \S}$ \\ Kenneth W. Dunn, ${ }^{\neq \S}$ Kevin S. Lorenz, ${ }^{\text {" }}$ \\ Edward J. Delp," Paul Salama," \\ Bruce A. Molitoris, ${ }^{\ddagger \star * *}$ and Irina Petrache ${ }^{\star \star * *}$ \\ From the Center for Immunobiology," and the Departments of \\ Anesthesia ${ }^{\dagger}$ and Medicine, ${ }^{\ddagger}$ Indiana University, Indianapolis; the \\ Indiana Center for Biological Microscopy, ${ }^{\circledR}$ Indianapolis; the \\ School of Electrical and Computer Engineering," Purdue \\ University, West Lafayette; the Department of Electrical and \\ Computer Engineering," Indiana University-Purdue University, \\ Indianapolis; and the Richard L. Roudebush Veterans Affairs \\ Medical Center, ${ }^{* *}$ Indianapolis, Indiana
}

Intravital microscopy has been recognized for its ability to make physiological measurements at cellular and subcellular levels while maintaining the complex natural microenvironment. Two-photon microscopy (TPM), using longer wavelengths than single-photon excitation, has extended intravital imaging deeper into tissues, with minimal phototoxicity. However, due to a relatively slow acquisition rate, TPM is especially sensitive to motion artifact, which presents a challenge when imaging tissues subject to respiratory and cardiac movement. Thoracoabdominal organs that cannot be exteriorized or immobilized during TPM have generally required the use of isolated, pump-perfused preparations. However, this approach entails significant alteration of normal physiology, such as a lack of neural inputs, increased vascular resistance, and leukocyte activation. We adapted techniques of intravital microscopy that permitted TPM of organs maintained within the thoracoabdominal cavity of living, breathing rats or mice. We obtained extended intravital TPM imaging of the intact lung, arguably the organ most susceptible to both respiratory and cardiac motion. Intravital TPM detected the development of lung microvascular endothelial activation manifested as increased leukocyte adhesion and plasma extravasation in response to oxidative stress inducers PMA or soluble cigarette smoke extract. The pulmonary microvasculature and alveoli in the intact animal were imaged with compa- rable detail and fidelity to those in pump-perfused animals, opening the possibility for TPM of other thoracoabdominal organs under physiological and pathophysiological conditions. (Am J Pathol 2011, 179: 75-82; DOI: 10.1016/j.ajpath.2011.03.048)

There is an increasing need for visualizing dynamic cellular and molecular events in the context of their natural complex tissue environment. This task has long been limited by artifacts generated during tissue fixation or by changes in cell behavior induced by ex vivo culture systems. With the recent development of a wide variety of fluorescent probes and transgenic expression of fluorescent labels, intravital microscopy allows the dissection of molecular events with subcellular resolution in near real time in the intact animal. Nevertheless, serious challenges remain. For example, the use of routine epifluorescence microscopy for intravital imaging is limited by a superficial plane of visualization and photodamage to the lipid- and water-rich structures of many biological tissues. Although confocal microscopy is capable of imaging structures at a deeper tissue level, the techniques used to eliminate light arising outside the focal point (eg, pinhole aperture) inevitably degrade the signal whereas the problem of phototoxicity remains. The development of two-photon excitation microscopy (TPM) has addressed some of these limitations. In contrast to epifluorescence and confocal microscopy, excitation of fluorescent probes in TPM results from the simultaneous collision of two long-wavelength photons with the fluorophore. Because excitation only occurs at the focal point, there is no out-of-plane fluorescence to reject, and a stronger signal

Supported by the National Institutes of Health (NIH)-National Heart, Lung and Blood Institute grants R01 HL 077328 (I.P.); R21 DA029249-01 (I.P. and R.G.P.); T32 (M.B.B.); and NIH O'Brien P-30 grant 5P30DK079312.

Accepted for publication March 29, 2011.

R.G.P. and M.B.B. contributed equally to this work

None of the authors disclosed any relevant financial relationships.

Supplemental material for this manuscript can be found at $h t t p: / / a j p$. amjpathol.org or at doi: 10.1016/j.jmoldx.2010.11.003.

Address reprint requests to: Robert G. Presson Jr, M.D., or Irina Petrache, M.D., Walther Hall-R3, C400, 980 W. Walnut Street, Indianapolis, IN 46202. E-mail: rpresson@iupui.edu or ipetrach@iupui.edu. 
can be acquired. Moreover, the lower-energy excitation photons in TPM are less likely to produce phototoxicity and are able to penetrate farther into tissue, thus permitting examination into organs of living animals. Unfortunately, the relatively slow rate of image acquisition makes TPM especially sensitive to motion artifact, which presents a major obstacle to imaging tissues subject to respiratory and cardiac movement. Motion artifact can be circumvented for organs, such as the kidney ${ }^{1}$ and liver, ${ }^{2}$ that can be surgically explanted from the body for imaging without alteration in perfusion or organ function. Thoracoabdominal organs that cannot be exteriorized or immobilized during TPM, such as the lung, have generally required the use of isolated, pump-perfused preparations. ${ }^{3-5}$ However, measurements obtained from isolated preparations may vary from the intact animal (that maintains tissue perfusion by means of a beating heart) for a number of reasons, including an absence of neural inputs, increased vascular resistance, and activation of leukocytes by foreign surfaces of the perfusion circuit. ${ }^{4}$ Motion can be largely eliminated in intact preparations for intravital imaging by suspending ventilation during acquisition, ${ }^{6}$ but the duration of apnea can generally not be long enough for image capture in more than one plane, given TPM's relatively slow framing rate. Suspending ventilation is also not an ideal solution to motion artifact in lung imaging because of potentially confounding effects introduced by altered blood gases and pulmonary and systemic pressures. Here, we describe novel adaptations of previously pioneered intravital microscopy techniques $^{4,7}$ that permit high-fidelity imaging of lung maintained within the thoracoabdominal cavity of a living, breathing animal for a prolonged period of time. Our work complements two very recent reports that also describe the innovative use of intravital lung TPM in intact rodent models. ${ }^{8,9}$ In addition, we present the first detailed methodology for management of motion artifact to obtain consistent, high-quality three-dimensional and time-lapse TPM imaging of murine lung under physiological conditions and document its superiority to the pump-perfused preparation in maintaining physiological cardiopulmonary hemodynamic and biochemical parameters.

\section{Materials and Methods}

\section{Stabilization at the Lung-Microscope Interface}

To perform TPM of the lung in the intact rat, we optimized the lung-microscope interface with an imaging window uniquely designed to minimize cardiac and respiratory motion while permitting the use of high numerical aperture TPM objectives (see Supplemental Figure S1 at http://ajp.amjpathol.org). The window was located in the center of a purpose-made acrylic tray (see Supplemental Figure S1A at $h$ ttp://ajp.amjpathol.org), constructed for intravital microscopy of organs within the thoracoabdominal cavity, that was mounted on the microscope stage of the TPM system. The window design consisted of a corrosion-resistant aluminum frame (see Supplemental Figure S1, B and C, at http://ajp.amjpathol.org) holding a circular coverslip $18 \mathrm{~mm}$ in diameter. The coverslip was surrounded by a vacuum ring that held the lung in contact with the coverslip and reduced respiratory movement, allowing observation of a $0.5 \mathrm{~cm}^{2}$ area of the overlying lung (see Supplemental Figure S1D at http:// ajp.amjpathol.org). A similar design was adapted to image the mouse lung.

\section{Intact Animal Preparation}

All animal studies were conducted in compliance with the Institutional Animal Care and Use Committee guidelines of Indiana University. Adult male Sprague Dawley rats (Harlan, Indianapolis, IN) weighing 250 to $350 \mathrm{~g}$ were anesthetized by inhaled isoflurane (5\% in oxygen) and orotracheally intubated with a 6-F catheter. The animals were placed on a servo-controlled heating pad, and the left carotid artery and right external jugular vein were cannulated via cutdowns. Surgical instruments were autoclaved before each use to minimize the introduction of foreign pathogens. The lungs were ventilated using a tidal volume of $6 \mathrm{~mL} / \mathrm{kg}$, a rate of 60 breaths/minute, an inspired $\mathrm{O}_{2}$ content of $100 \%$, and isoflurane to maintain general anesthesia. Airway pressure and esophageal temperature were monitored and maintained continuously at 14 to $19 \mathrm{~cm} \mathrm{H}_{2} \mathrm{O}$ (peak), and 36 to $37{ }^{\circ} \mathrm{C}$, respectively. End-expiratory pressure was maintained at $\sim 5 \mathrm{~cm} \mathrm{H}_{2} \mathrm{O}$. Blood gases were sampled periodically, respiratory rate was adjusted to maintain a $\mathrm{pACO}_{2}$ of 35 to 40 torr, and inspired oxygen concentration was adjusted to maintain a $\mathrm{pAO}_{2}>90$ torr. The systemic arterial pressure was monitored continuously, and lactated Ringer's solution was infused via the venous catheter at a maintenance rate of $3 \mathrm{~mL} / \mathrm{kg} / \mathrm{hour}$ with boluses given as needed to replace blood loss from sampling. The animals were placed in the right lateral decubitus position, and a 6 -cm-long skin incision was made $2 \mathrm{~cm}$ caudal to the posterior edge of the left scapula. The latissimus dorsi was retracted posteriorly, and a thoracotomy was made in the left fifth intercostal space. Stay sutures were placed around the anterior and posterior ends of the fifth and sixth ribs approximately $2 \mathrm{~cm}$ apart (see Supplemental Figure S2 at $h$ ttp://ajp.amjpathol.org). The thoracotomy was held open by tension on the stay sutures, and the animals were placed in the left lateral decubitus position with the left lung centered over the window. End-expiratory pressure was briefly increased to 10 to $20 \mathrm{~cm} \mathrm{H}_{2} \mathrm{O}$, which brought the lung into contact with the coverslip. $\mathrm{A}$ vacuum $(-40 \mathrm{~mm} \mathrm{Hg})$ was applied to the window that held the lung in position after the end-expiratory pressure was returned to $5 \mathrm{~cm} \mathrm{H}_{2} \mathrm{O}$, a level typically used for mechanical ventilation, which does not adversely affect microcirculation. ${ }^{10} \mathrm{~A}$ similar procedure was performed in mice with the following modifications: Mice were orotracheally intubated with a 20-gauge catheter and ventilated at a rate of 130 breaths/minute. The right internal jugular vein was cannulated via cutdown with a 26-gauge catheter for administration of fluid and fluorescent probes. A thoracotomy was performed in the fifth left intercostal space, and the sixth rib was excised. The window, mea- 
suring $1 \mathrm{~cm}$ in diameter, was interfaced with the lung via this thoracotomy, as described for the rat.

\section{Pump-Perfused Lung Preparation}

Because respiration can be suspended for long periods without altering blood gases and because there is no cardiac motion, we also performed TPM imaging of the lung in the isolated pump-perfused preparation for comparison to the images acquired from the intact rat. Surgical procedures for the pump-perfused lung preparation were similar to the procedures for the intact preparation with a few exceptions. For the pump-perfused preparation, following orotracheal cannulation, carotid cannulation, and exsanguination, the blood was added to a perfusion circuit already primed with buffer $(20 \mathrm{mmol} / \mathrm{L}$ HEPES, $125 \mathrm{mmol} / \mathrm{L} \mathrm{NaCl}, 3.5 \mathrm{mmol} / \mathrm{L} \mathrm{KCl}, 1 \mathrm{mmol} / \mathrm{L}$ $\mathrm{CaCl}_{2}, 1 \mathrm{mmol} / \mathrm{L} \mathrm{MgCl}, 5 \mathrm{mmol} / \mathrm{L}$ glucose, and $1 \%$ fetal bovine serum, final hematocrit $\sim 10 \%$, circulating volume $\sim 30 \mathrm{~mL}$ ). The left chest wall was excised, and the apex of the heart was transected. The arterial perfusion cannula was passed through the right ventricle across the pulmonic valve into the main pulmonary artery and was secured with a ligature passed around the main pulmonary artery and aortic root. The venous perfusion cannula was passed through the left ventricle across the mitral valve into the left atrium and was secured with a ligature around the atrioventricular groove. Ventilation was resumed with $5 \% \mathrm{CO}_{2}$ in air, and perfusion was initiated at a flow rate of $5 \mathrm{~mL} / \mathrm{min}$. Blood was pumped (Gilson Minipuls 3; Gilson, Middleton, WI) through a heat exchanger into the pulmonary artery and drained passively from the left atrium into a reservoir. Pump flow rate was slowly increased to maintain a mean arterial pressure $<15 \mathrm{~mm}$ $\mathrm{Hg}$, and the height of the reservoir was adjusted to obtain a venous pressure of $\sim 2 \mathrm{~mm} \mathrm{Hg}$. Animal positioning and the regulation of blood gases, esophageal temperature, and pressures for intravital microscopy were performed as described for the intact preparation.

\section{Fluorescent Conjugates}

Rat serum albumin (RSA; Sigma-Aldrich, St. Louis, MO) or a 150-kDa amino dextran (TdB Consultancy, Uppsala, Sweden) was conjugated to fluorescein isothiocyanate (FITC) or Texas Red (Invitrogen, Carlsbad, CA) as outlined in the manufacturer's technical instructions for amine reactive probes (Molecular Probes/Invitrogen). After conjugation of FITC or Texas Red to the RSA, any unbound dye molecules were removed by dialyzing against $0.9 \% \mathrm{NaCl}$ in double-distilled $\mathrm{H}_{2} \mathrm{O}$ using a 300$\mathrm{kDa}$ cutoff MWCO filter (Cellulose Ester Membrane from Spectrum Laboratories, Rancho Dominguez, CA). Either FITC-RSA (12 to $14 \mathrm{mg} / \mathrm{kg}$ ) or similarly labeled FITC- or Texas Red-Dextran (150 kDa amino dextran; 20 to 22 $\mathrm{mg} / \mathrm{kg}$ ) were administered intravenously (i.v.) to label the circulating plasma. Nuclei were stained with Hoechst 33258 (10 to $12 \mathrm{mg} / \mathrm{kg}$; i.v.; Invitrogen), and leukocytes were labeled with Rho-G6 $(0.2 \mathrm{mmol} / \mathrm{L}$ in saline; $0.3 \mathrm{~mL} /$ kg; i.v.; Sigma-Aldrich).

\section{Additional Motion Reduction Techniques}

Although the vacuum ring markedly reduced respiratory and cardiac-induced lung motion, in some cases, additional procedures were needed to obtain high-fidelity images without interruption of ventilation. Therefore, we developed two approaches to deal with these challenges: gated imaging and frame registration. In gated imaging, Z-stack acquisition is synchronized (gated) to the respiratory cycle for "between breath" image capture. Gated imaging was accomplished by communication between a laptop computer running LabVIEW software (National Instruments Corporation, Austin, TX) that controlled the ventilator and a second computer running the imaging software (Fluoview 2.1c; Olympus, Center Valley, PA) via a DB15 interface. Briefly, the ventilator was modified to send a 5-V signal to the laptop computer at the start of expiration. At that point, the laptop paused the ventilator via a relay and then signaled the imaging computer to start a scan. After completion of the scan ( $\sim 2$ seconds), a signal was sent to the laptop that closed the relay, resuming ventilation. This cycle was repeated until the desired number of scans was obtained.

Due to a lower frequency of image acquisition, gating inherently decreases temporal resolution. Therefore, to obtain high-fidelity images at high speed when cardiac and respiratory motion was not sufficiently reduced by the vacuum window, a novel frame registration method was applied in image processing. Motion artifacts were reduced postcollection using a novel nonrigid image registration method that uses B-splines to correct for motion artifacts occurring in images collected in time series or in three-dimensional volumes. The method involves creating a uniform grid of control points in the images of the time series, each of which is manipulated by optimizing a cost function based on image similarity and grid smoothness. The technique is described more fully in Lorenz et al. ${ }^{11}$

\section{Two-Photon Microscopy System}

Unless otherwise specified, TPM was conducted on a Bio-Rad MRC 1024 confocal/two-photon system (BioRad, Hercules, CA) mounted on a vibration-isolation table (see Supplemental Figure S3 at http://ajp.amjpathol.org). The illumination source was a tunable Tsunami Ti:Sapphire laser (Spectra-Physics, Mountain View, CA). The microscope was an inverted Nikon Eclipse TE200 (Nikon, Melville, NY). The band-pass filters used for the red and green channels were 605/690 nm and 525/550 nm, respectively. The excitation wavelength was set at $800 \mathrm{~nm}$ for all experiments. Either a $\times 60$ or a $\times 20$ Nikon Plan Apo water immersion objective (NA 1.2 and 0.8) was used for imaging and was warmed with an objective heater (Warner Instruments, Hamden, CT). All image series were collected at a constant pixel dwell time, which yielded a 1.1-second frame time for the frame size of $512 \times 512$ pixels $(169 \times 169 \mu \mathrm{m})$. 


\section{Cigarette Smoke Soluble Extract}

Filtered research-grade cigarettes (1R3F) from the Kentucky Tobacco Research and Development Center (University of Kentucky, Lexington, KY) were used for preparing aqueous cigarette smoke extract. Cigarette smoke (100\%) was prepared by bubbling smoke from four cigarettes into $5 \mathrm{~mL}$ of PBS at a rate of one cigarette/minute to $0.5 \mathrm{~cm}$ above the filter, ${ }^{12}$ followed by $\mathrm{pH}$ adjustment to 7.4 and $0.2-\mu \mathrm{m}$ filtration.

\section{Measures of Microvascular Flow and Leukocyte Adhesion}

Microvascular flow was determined for horizontally oriented vessels as previously described ${ }^{13}$ using vessel diameter and blood velocity as calculated by slope of the black streak that appears where red blood cells are imaged in motion. Stationary Rho-G6-labeled intraluminal leukocytes adherent to the microcirculation within a single field were counted using frames of time-series movies, as previously described. ${ }^{14}$

\section{Statistical Analyses}

Statistical analyses were performed using SigmaStat 3.5. Comparisons among groups were made using analysis of variance followed by multiple comparisons versus a control group (Holm-Sidak method). For experiments in which two conditions were being compared, a two-tailed Student's $t$-test was used. All experiments were performed at least three times. All data are expressed as mean \pm SEM, and statistically significant differences were considered if $P<0.05$.

\section{Results}

\section{TPM Imaging of the Lung}

Mild suction incorporated into a custom-made imaging window effectively eliminated cardiac and respiratory motion at the lung-microscope interface in most intact preparations for high-quality TPM. Single-frame images from time-series acquisition movies in the intact rat under physiological conditions (during ventilation, heart perfused) are shown in Figure 1, illustrating the three main vessel types captured by TPM: pulmonary arterioles, venules, and capillaries. For both the intact rat and the intact mouse, intravenous nuclear staining revealed primarily endothelial cells lining the vessels in images obtained by Z-stack (see Supplemental Video S1 in rat and Video S2 in mouse at http://ajp.amjpathol.org) or timeseries acquisition (see Supplemental Video S3 in rat and Video S4 in mouse at http://ajp.amjpathol.org). When the intravenous administration was combined with intratracheal delivery, the nuclear dye penetrated deep in the parenchyma, providing staining of additional nuclei, seen in the three-dimensional reconstruction in Supplemental Figure S4 (available at $h$ ttp://ajp.amjpathol.org). Based on location and size, these additional nuclei were likely con-
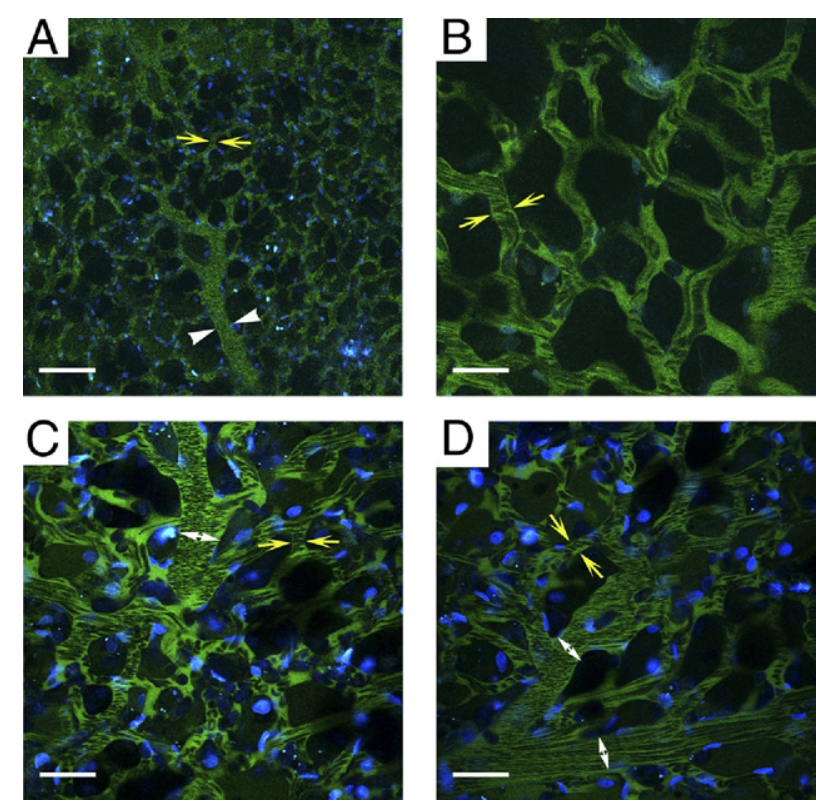

Figure 1. Representative frames of time-series TPM images in the intact rat showing FITC-labeled (green) pulmonary microvasculature in low magnification $\times 20(\mathbf{A})$ and high magnification $\times 60(\mathbf{B}-\mathbf{D})$. Note detailed microvasculature morphology of medium- and small-sized arterioles (white arrowheads, $\mathbf{A}$ ), venules (white bidirectional arrows, $\mathbf{C}$ and $\mathbf{D}$ ), and capillaries (yellow arrows) surrounding normal alveolar airspaces. Nuclei are stained with intravenous Hoechst (blue), and circulating cells appear as black streaks within the vessels. Scale bars: $75 \mu \mathrm{m}(\mathbf{A})$ and $25 \mu \mathrm{m}(\mathbf{B}-\mathbf{D})$.

tained within alveolar epithelial cells and alveolar macrophages.

Images from the intact animal were equal in detail and fidelity to those obtained for comparison in the pumpperfused preparation, in which respiratory and cardiac motion is not a factor (Figure 2). In either the intact animal or the pump-perfused preparation, the average number of alveolar spaces captured in a single field of view was $38 \pm 4$ (with the $\times 60$ objective) or $62 \pm 8$ (with the $\times 20$ objective), at a maximum depth ranging from 40 to $60 \mu \mathrm{m}$ from the pleural surface.

\section{Gating Imaging and Frame Registration}

In some cases, where lung-microscope stabilization provided by the vacuum ring could not completely eliminate respiratory and cardiac-induced lung motion, gated imaging or frame registration was additionally used. Gated imaging was used for Z-stack acquisition and entailed computerized synchronization of the respiratory cycle to image capture, effectively eliminating motion and maximizing clarity of anatomical detail in single-plane and reconstructed three-dimensional images (Figure 3). Although the gating algorithm decreased the overall respiratory rate, it did not significantly change the arterial blood gases (see Supplemental Table S1 at http://ajp. amjpathol.org). For time-series collections, frame registration ${ }^{11}$ was used in postcapture image processing to correct for motion artifact when necessary and effectively removed the rhythmic lung motion that was captured in some movies acquired during ventilation, without com- 

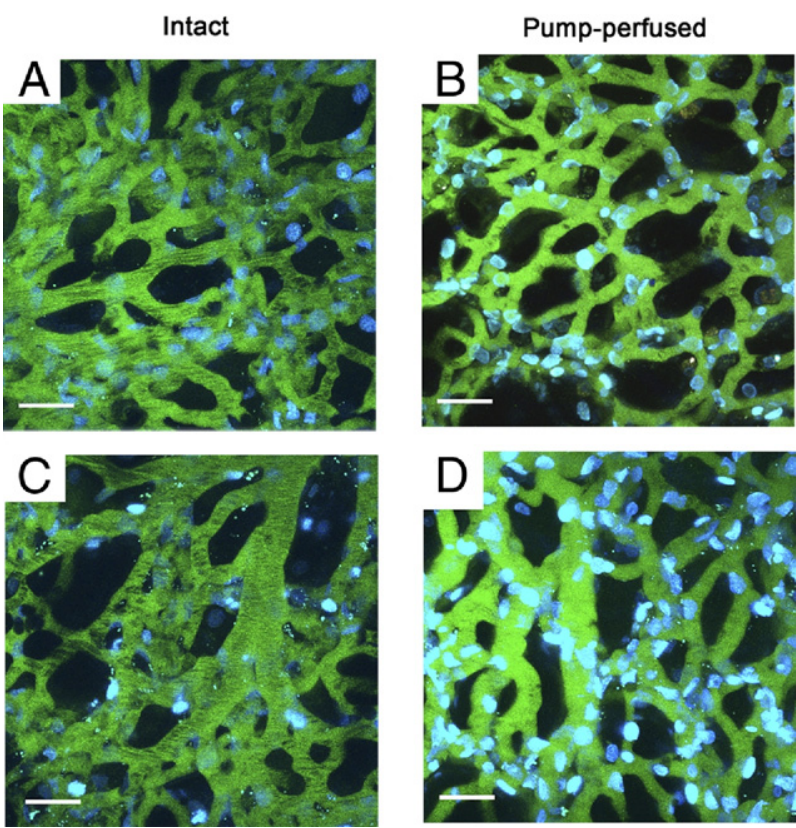

Figure 2. Three-dimensional reconstruction of FITC-labeled microvasculature (green) surrounding normal alveolar airspaces (dark regions) imaged in intact preparations (A and $\mathbf{C}$ ) are comparable to pump-perfused preparations $(\mathbf{B}$ and $\mathbf{D})$. Nuclei are stained blue with intravenous Hoechst. Scale bars $=25$ $\mu \mathrm{m}$. Note that the capillary circulation $(\mathbf{A}-\mathbf{D})$ and larger venules $(\mathbf{C}$ and $\mathbf{D})$ are visualized by TPM with similar detail in the two preparations.

promising temporal resolution (see Supplemental Video S3 at $h$ ttp://ajp.amjpathol.org).

\section{Quality of the Physiology}

Intact rats were hemodynamically stable during experiments, which averaged 6 to 7 hours, from the time of
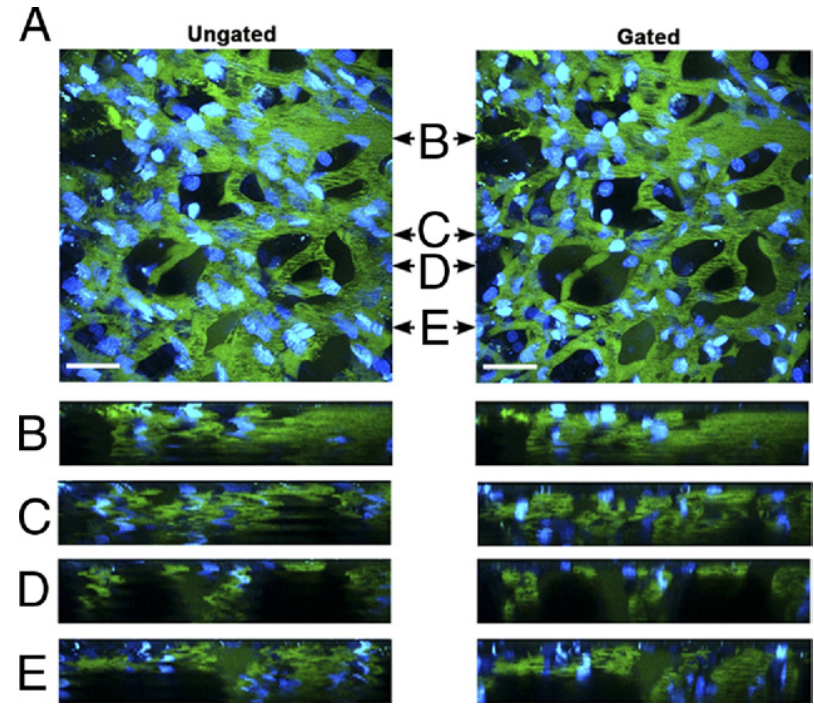

Figure 3. Gated imaging eliminates motion for maximum clarity in threedimensional TPM reconstructions. A: Comparison between ungated (left) and gated (right) image acquisition of an identical field of view showing FITC-labeled (green) alveolar microvasculature in the intact rat. Reconstructions in the $x-z$ orientation correspond to the indicated slice regions $(\mathbf{B}-\mathbf{E})$ from the 3 dimensional images. Nuclei are stained with intravenous Hoechst (blue). Scale bars $=25 \mu \mathrm{m}$.
Table 1. Vital Signs and Arterial Blood Parameters during TPM in the Intact Animal

$\begin{array}{lc}\text { Systolic arterial pressure, } \mathrm{mm} \mathrm{Hg} & 100 \pm 8 \\ \text { Diastolic arterial pressure, } \mathrm{mm} \mathrm{Hg} & 62 \pm 12 \\ \text { Heart rate, beats/minute } & 350 \pm 44 \\ \text { Arterial } \mathrm{pCO}_{2}, \mathrm{~mm} \mathrm{Hg} & 42 \pm 8 \\ \text { Arterial } \mathrm{pO}_{2}, \mathrm{~mm} \mathrm{Hg} & 110 \pm 18 \\ \text { Arterial } \mathrm{pH} & 7.42 \pm 0.04 \\ \text { Hematocrit, \%PCV } & 34 \pm 6 \\ \text { Hemoglobin, g/dL } & 12 \pm 2\end{array}$

These values are within physiological range and are presented as mean $\pm \mathrm{SD} ; n=11$

PCV, packed cell volume; TPM, two-photon microscopy.

initiation of surgical preparation to placement on the microscope (2 to 3 hours), to the completion of intravital imaging (3 to 4 hours). Peak arterial blood pressure was maintained above $90 \mathrm{~mm} \mathrm{Hg}$; and mean partial pressures of oxygen $\left(\mathrm{pAO}_{2}\right)$ and carbon dioxide $\left(\mathrm{pACO}_{2}\right)$ were maintained in the range of 90 to $100 \mathrm{~mm} \mathrm{Hg}$ (fraction of inspired $\mathrm{O}_{2}, 0.21$ to 0.30 ) and 35 to $45 \mathrm{~mm} \mathrm{Hg}$, respectively (Table 1). Vessel diameter and microvascular flow were stable over the period of observation (see Supplemental Table S2 at http://ajp.amjpathol.org). Further, microvascular flow was better maintained in pulmonary arterioles, venules, and capillaries of the intact animal compared to those imaged in the in the pump-perfused lung (Table 2). The significantly lower microvascular flow in the pump-perfused lung is due to the elevated pulmonary vascular resistance characteristic of this preparation. ${ }^{15}$ This necessitated a low pump flow rate (to maintain perfusion pressures in the normal range), even with a hemoglobin concentration of $4 \mathrm{~g} / \mathrm{dL}$. In aggregate, these data show that physiological parameters were maintained over an extended period of experimental observation without tissue damage in the intact rat preparation.

\section{Applications to Studies of Lung Pathology}

The novel adaptation of intravital imaging methods described above can be used to investigate in vivo organ physiology, signaling, pharmacodynamics, and disease

Table 2. Microvasculature Hemodynamic Parameters in the Intact (Live) Animal Compared to Those of the Pump-Perfused Preparation during Intravital TPM

\begin{tabular}{|c|c|c|}
\hline & \multicolumn{2}{|c|}{ Flow (mm³/second) } \\
\hline & $\begin{array}{l}\text { Intact prep } \\
\quad(n=11)\end{array}$ & $\begin{array}{l}\text { Pump-perfused } \\
\text { preparation } \\
(n=6)\end{array}$ \\
\hline $\begin{array}{l}\text { Medium arteriole } \\
\quad(19-31 \mu \mathrm{m})\end{array}$ & $580 \pm 300$ & $430 \pm 170$ \\
\hline Small arteriole (10-18 $\mu \mathrm{m})$ & $140 \pm 90^{*}$ & $40 \pm 9$ \\
\hline Medium venule (19-28 $\mu \mathrm{m})$ & $640 \pm 380^{\star *}$ & $200 \pm 90$ \\
\hline Small venule (10-18 $\mu \mathrm{m})$ & $150 \pm 80^{\star * *}$ & $85 \pm 48$ \\
\hline Capillary $(6-9 \mu \mathrm{m})$ & $31 \pm 16^{\star \star \star}$ & $19 \pm 8$ \\
\hline
\end{tabular}

Values are the mean $\pm S D$ of hemodynamic parameters recorded during 3 hours of experiments.

${ }^{\star} P<0.05,{ }^{* \star} P<0.01$, and ${ }^{* \star *} P<0.001$; all versus pump-perfused preparation.

TPM, two-photon microscopy 


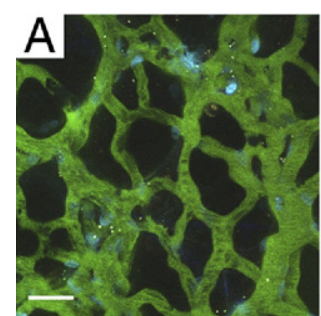

$0 \mathrm{~min}$

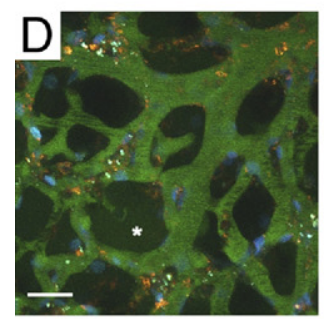

$9 \mathrm{~min}$

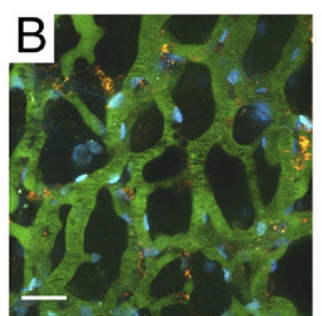

$0 \mathrm{~min}$

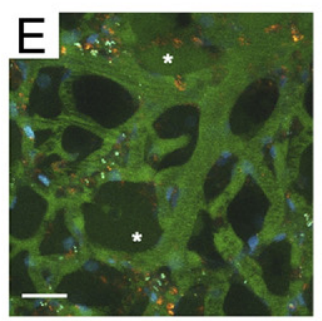

$12 \min$

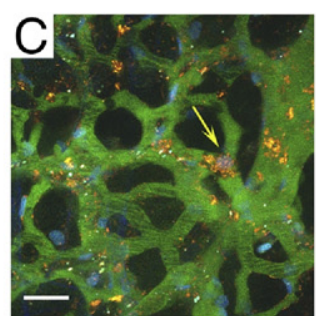

$5 \mathrm{~min}$

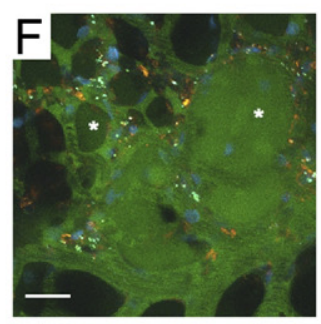

$30 \mathrm{~min}$

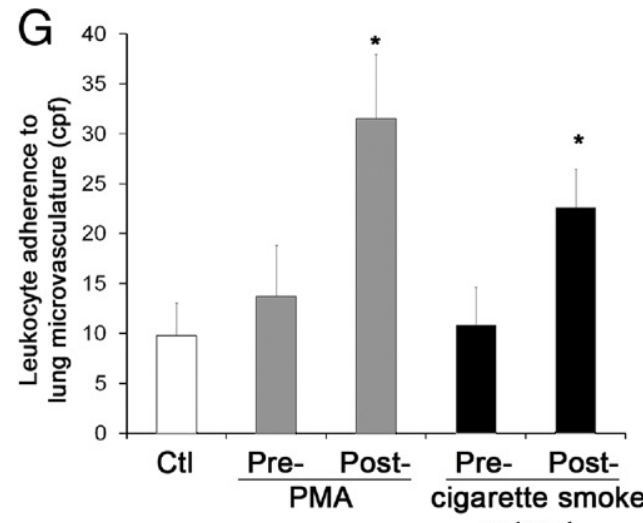

extract

Figure 4. Capturing real-time development of pulmonary edema using TPM of the lung microvasculature in the intact rat. Three-dimensional reconstruction of FITC-labeled vessels (green) surrounding alveoli without (A) and with Rho-6G-labeled leukocytes (red, B-F) imaged before (A and B) and after (C-F) intravenous administration of PMA $(1 \mathrm{mmol} / \mathrm{L}, 0.10 \mathrm{mg} / \mathrm{kg})$. Nuclei are stained with intravenously administered Hoechst (blue). Note increasing leukocyte sequestration in the capillaries at 5 minutes (C; arrow) and plasma extravasation (asterisks) into airspaces at 9 minutes (D), 12 minutes (E), and 30 minutes (D) post-PMA administration. D and $\mathbf{E}$ depict an identical field of view. Scale bars $=25 \mu \mathrm{m}$. Representative image of $n=3$ animals. G: Quantification of leukocyte adherence in vivo in response to PMA ( $1 \mathrm{mmol} / \mathrm{L}$ in dimethylformamine, $100 \mu \mathrm{L} / \mathrm{kg}$, i.v; 5 to 20 minutes) or soluble cigarette smoke extract ( $100 \%$, $2 \mathrm{~mL} / \mathrm{kg}$ i.v; 20 to 30 minutes) administration compared to untreated animals ( $n=2$ rats). Mean $\pm \mathrm{SD} ;{ }^{*} P<0.001$ versus control; $n=5$ to 19 time-lapse movies.

pathogenesis. For example, we captured the dynamic responses of the lung microvasculature to perturbations induced by the intravenous administration of the oxidative stress and tumor promoter 4b-phorbol 12-myristate 13-acetate (PMA; Santa Cruz Biotechnology, Santa Cruz, $\mathrm{CA} ; 1 \mathrm{mmol} / \mathrm{L}$ in dimethylformamine). PMA is known to activate lung macrophages and result in irreversible parenchymal damage via destruction of endothelial and alveolar epithelial cells of the distal airways. Breach in alveolar and microvasculature barrier function from PMA administration results in alveolar hemorrhage and flooding. ${ }^{16}$ In these experiments, intravenous rhodamine $6 \mathrm{G}$ (Rho-6G; 0.5 mg/kg) was used to label leukocytes for fluorescence imaging (Figure 4; see also Supplemental Video S5 at http://ajp.amjpathol.org). In response to PMA administration, leukocytes accumulated in the lung parenchyma within 5 minutes and demonstrated reduced mobility through the microcirculation, suggesting increased adhesion to the endothelium (Figure 4C; see also Supplemental Video S6 at http://ajp.amjpathol.org). In addition, we recorded progressive breaching of the lung microvascular barrier signaled by extravasation of FITCRSA into the alveolar airspaces (Figure 4, D-F; see also Supplemental Video S6 at http://ajp.amjpathol.org), consistent with the development of pulmonary edema. The early development of endothelial activation, manifested as increased leukocyte adhesion to the microvasculature can be quantified using a technique we previously applied for the kidney microcirculation. ${ }^{14}$ We noted a significant increase in leukocyte adhesion starting at 5 minutes following PMA administration (Figure 4). A similar effect was induced by administration of soluble components of cigarette smoke (Figure 4), known to induce oxidative stress by highly diffusible free radicals that cause microvascular activation in vitro (Wagner ${ }^{17}$ and unpublished data).

\section{Discussion}

We have described an innovative method of intravital microscopy that permits high-resolution subsurface imaging of organs within the thoracoabdominal cavity of a living animal without interference from cardiac or respiratory motion. First, we used a vacuum ring imaging window, custom-designed for the TPM optics that physically restrained tissue at the organ-microscope interface. Second, we applied gated imaging or frame registration as adjunctive approaches to optimize image quality for situations in which the vacuum ring alone did not sufficiently immobilize the observed tissue. The method of gating imaging to the respiratory cycle enabled capture of a series of planes in the z-direction "between breaths" for use in three-dimensional image reconstructions. Alternatively, frame registration applied a postacquisition algorithm to correct for rhythmic respiratory motion when maximal temporal resolution was required. These highly detailed, high-quality and stable TPM images of the lung, an organ inexorably coupled to both cardiac and respiratory movement, obtained in physiological conditions, are evidence of the power of these techniques for imaging thoracoabdominal organs in situ. Early attempts to deal with the problem of motion during intravital imaging of the lung in the intact animal included simply suspending respiration, ${ }^{6}$ with observations made during apnea. Major progress toward physiological imaging of the lung was made by Wagner, ${ }^{7}$ who implanted a device in the chest wall containing a central 
window and a surrounding vacuum manifold that arrested cardiorespiratory motion in the observation field during serial measurements. Although this approach permitted bright-field imaging of the subpleural microcirculation in a living, breathing animal, observations were limited to less than a few microns from the surface. Later, fluorescence microscopy was applied using a similar window, ${ }^{18}$ and conventional confocal microscopy was used to visualize alveoli deeper than those accessible to wide-field microscopy. ${ }^{19,20}$ However, these approaches were performed only in isolated (pump-perfused) lung preparations. Recently, the use of a probe inserted transdiaphragmatically or transthoracically allowed the use of the confocal microscopy in the intact animal. ${ }^{21}$ However, the confocal aperture's rejection of scattered light leads to a relatively weak signal that may render a poor image quality, and no quantitative or dynamic time-lapse analyses during the ventilatory motion were reported. With TPM, there is no out-of-plane fluorescence to reject, and therefore, no confocal aperture to obstruct photon collection. The resulting signal is stronger, as shown in a pumpperfused preparation, ${ }^{4}$ and in our hands, was capable of imaging structures as deep as $80 \mu \mathrm{m}$ into the lung with good resolution. Within these parameters, we demonstrated that both alveolar capillary structures and larger venules and arterioles can be adequately visualized in real time while maintaining physiological parameters for over 3 hours of study. The quality of the preparation and its performance surpassed that of the pump-perfused preparation, while simplifying and shortening the surgical preparation time and trauma to the animal. Although measures were taken to minimize contamination by foreign pathogens during each animal preparation, a potential risk of any surgery as invasive as required for intravital imaging within the thorax is that nonsterile conditions may affect leukocyte behavior at the imaging site or other endpoints of analysis. Although our control imaging studies of the lung microcirculation over the entire duration of the experiment (up to 3 hours) did not reveal marked increases in polymorphonuclear leukocyte accumulation or adherence to the microendothelium, our method can be further improved by ensuring total sterility of the surgical field and of the animal-microscope interface. Two independent reports of intravital TPM to investigate lung inflammation in rodents ${ }^{8,9}$ have been published in the past months, highlighting the importance and emergence of this method for the study of complex integrated responses in the lung. To complement these studies, we provide a detailed and reproducible protocol of intravital TPM of the lung that generates detailed, stable, highfidelity three-dimensional and time-lapse imaging of the murine lung microcirculation and alveolar spaces; we apply for the first time noninvasive approaches to stabilize the intravital tissue imaging (avoiding the use of superglue or breath-hold to immobilize the lung to the imaging window); and we document the preservation of functional parameters such as cardiopulmonary hemodynamics and biochemical homeostasis in the animal for the duration of the experiment (up to 3 hours).

The adapted intravital methods described herein have a vast breadth of application within medical research, particularly for real-time visualization of processes within thoracoabdominal organs without significant disruption of the normal physiological milieu. We demonstrated a rapid and sensitive capture of intra-alveolar edema development and leukocyte sequestration in pulmonary capillaries following infusion of PMA, a typical oxidative stress inducer. Leukocyte adhesion could be quantified and allowed sensitive detection of endothelial cell activation, consistent with early inflammatory changes in response to both PMA and exposure to soluble components of cigarette smoke. Such changes could not be accurately detected in pump-perfused preparations, which are known to activate leukocytes by exposure to foreign surfaces. We also used our imaging technique to calculate blood flow in various pulmonary vascular structures. These are just a few examples of applications of our method to study tissue physiology and pathology, which could be expanded to include studies of vasoreactivity ${ }^{22}$; endothelial or epithelial permeability ${ }^{23,24}$; leukocyte adherence and rolling ${ }^{8,9,14}$; cell injury and apoptosis ${ }^{25}$; endo-, trans-, and exocytosis $^{26,27}$; ionic gradients ${ }^{28}$; metabolic status ${ }^{29}$; intracelIular trafficking and subcellular localization ${ }^{26}$; and drug deposition and biodistribution. ${ }^{30,31}$

Continued development of improved probes amenable to visualization of rapid events in living animals will advance the application of TPM to investigations of complex processes such as intra- and intercellular signal transduction. These mechanistic studies will be further facilitated by the use of genetically modified mice that use transgenically expressed functional probes. In conclusion, we describe a novel method of TPM of the lung parenchyma in a live rodent, which can be applied to any organ prone to motion artifact to study physiological and pathological processes in their natural, unperturbed, and interconnected environment.

\section{Acknowledgments}

We thank George Rhodes, M.D., for technical assistance with the microscope, and Jason Byars, who provided assistance with programming for gated imaging.

\section{References}

1. Dunn KW, Sandoval RM, Kelly KJ, Dagher PC, Tanner GA, Atkinson SJ, Bacallao RL, Molitoris BA: Functional studies of the kidney of living animals using multicolor two-photon microscopy. Am J Physiol Cell Physiol 2002, 283:C905-C916

2. Takeichi T, Engelmann G, Mocevicius P, Schmidt J, Ryschich E: 4-dimensional intravital microscopy: a new model for studies of leukocyte recruitment and migration in hepatocellular cancer in mice. $J$ Gastrointest Surg 2010, 14:867-872

3. Brueckl C, Kaestle S, Kerem A, Habazettl H, Krombach F, Kuppe H, Kuebler WM: Hyperoxia-induced reactive oxygen species formation in pulmonary capillary endothelial cells in situ. Am J Respir Cell Mol Biol 2006, 34:453-463

4. Kuebler WM, Parthasarathi K, Lindert J, Bhattacharya J: Real-time lung microscopy. J Appl Physiol 2007, 102:1255-1264

5. St. Croix CM, Leelavanichkul K, Watkins SC: Intravital fluorescence microscopy in pulmonary research. Adv Drug Deliv Rev 2006, 58 : $834-840$

6. Wearn JT, Ernstene AC, Bromer AW, Barr JS, German WJ, Zschiesche LJ: The normal behavior of the pulmonary blood vessels with 
observations on the intermittence of the flow of blood in the arterioles and capillaries. Am J Physiol 1934, 109:236-256

7. Wagner WW Jr: Pulmonary microcirculatory observations in vivo under physiological conditions, J Appl Physiol 1969, 26:375-377

8. Kreisel D, Nava RG, Li W, Zinselmeyer BH, Wang B, Lai J, Pless R, Gelman AE, Krupnick AS, Miller MJ: In vivo two-photon imaging reveals monocyte-dependent neutrophil extravasation during pulmonary inflammation, Proc Natl Acad Sci U S A 2010, 107:18073-18078

9. Looney MR, Thornton EE, Sen D, Lamm WJ, Glenny RW, Krummel MF: Stabilized imaging of immune surveillance in the mouse lung, Nat Methods 2011, 8:91-96

10. Lim LH, Wagner EM: Airway distension promotes leukocyte recruitment in rat tracheal circulation. Am J Respir Crit Care Med 2003, 168:1068-1074

11. Lorenz K, Salama P, Dunn K, Delp E: Non-rigid registration of multiphoton microscopy images using b-splines. Proc SPIE 2011, (in press)

12. Carp $\mathrm{H}$, Janoff $\mathrm{A}$ : Inactivation of bronchial mucous proteinase inhibitor by cigarette smoke and phagocyte-derived oxidants. Exp Lung Res 1980, 1:225-237

13. Kang JJ, Toma I, Sipos A, McCulloch F, Peti-Peterdi J: Quantitative imaging of basic functions in renal (patho)physiology. Am J Physiol Renal Physiol 2006, 291:F495-F502

14. Sharfuddin AA, Sandoval RM, Berg DT, McDougal GE, Campos SB, Phillips CL, Jones BE, Gupta A, Grinnell BW, Molitoris BA: Soluble thrombomodulin protects ischemic kidneys. J Am Soc Nephrol 2009, 20:524-534

15. Presson RG Jr, Todoran TM, De Witt BJ, McMurtry IF, Wagner WW Jr: Capillary recruitment and transit time in the rat lung. $J$ Appl Physiol 1997, 83:543-549

16. Ward PA: Oxidative stress: acute and progressive lung injury. Ann N Y Acad Sci 2010, 1203:53-59

17. Low B, Liang M, Fu J: p38 mitogen-activated protein kinase mediates sidestream cigarette smoke-induced endothelial permeability. J Pharmacol Sci 2007, 104:225-231

18. Lamm WJ, Bernard SL, Wagner WW Jr, Glenny RW: Intravital microscopic observations of 15 -microm microspheres lodging in the pulmonary microcirculation. J Appl Physiol 2005, 98:2242-2248

19. Safdar Z, Wang P, Ichimura H, Issekutz AC, Quadri S, Bhattacharya $\mathrm{J}$ : Hyperosmolarity enhances the lung capillary barrier. J Clin Invest 2003, 112:1541-1549
20. Carter EP, Matthay MA, Farinas J, Verkman AS: Transalveolar osmotic and diffusional water permeability in intact mouse lung measured by a novel surface fluorescence method. J Gen Physiol 1996, 108:133-142

21. Chagnon F, Fournier C, Charette PG, Moleski L, Payet MD, Dobbs LG, Lesur O: In vivo intravital endoscopic confocal fluorescence microscopy of normal and acutely injured rat lungs. Lab Invest 2010, 90:824-834

22. Bertuglia S: Intermittent hypoxia modulates nitric oxide-dependent vasodilation and capillary perfusion during ischemia-reperfusion-induced damage. Am J Physiol Heart Circ Physiol 2008, 294:H1914H1922

23. Dunn KW, Sandoval RM, Molitoris BA: Intravital imaging of the kidney using multiparameter multiphoton microscopy. Nephron Exp Nephrol 2003, 94:e7-e11

24. Oschatz C, Maas C, Lecher B, Jansen T, Bjorkqvist J, Tradler T, Sedlmeier R, Burfeind P, Cichon S, Hammerschmidt S, Muller-Esterl W, Wuillemin WA, Nilsson G, Renne T: Mast cells increase vascular permeability by heparin-initiated bradykinin formation in vivo. Immunity 2011, 34:258-268

25. Imamura R, Isaka Y, Sandoval RM, Ori A, Adamsky S, Feinstein E, Molitoris BA, Takahara S: Intravital two-photon microscopy assessment of renal protection efficacy of siRNA for p53 in experimental rat kidney transplantation models. Cell Transplant 2010, 19:1659-1670

26. Masedunskas A, Weigert R: Intravital two-photon microscopy for studying the uptake and trafficking of fluorescently conjugated molecules in live rodents. Traffic 2008, 9:1801-1810

27. Sandoval RM, Molitoris BA: Quantifying endocytosis in vivo using intravital two-photon microscopy. Methods Mol Biol 2008, 440:389-402

28. Brekke JF, Jackson WF, Segal SS: Arteriolar smooth muscle Ca2+ dynamics during blood flow control in hamster cheek pouch. J Appl Physiol 2006, 101:307-315

29. Mayevsky A, Barbiro-Michaely E: Use of NADH fluorescence to determine mitochondrial function in vivo. Int J Biochem Cell Biol 2009, 41:1977-1988

30. Amornphimoltham P, Masedunskas A, Weigert R: Intravital microscopy as a tool to study drug delivery in preclinical studies, Adv Drug Deliv Rev 2011, 63:119-128

31. van Lummel M, van Blitterswijk WJ, Vink SR, Veldman RJ, van der Valk MA, Schipper D, Dicheva BM, Eggermont AM, ten Hagen TL, Verheij M, Koning GA: Enriching lipid nanovesicles with short-chain glucosylceramide improves doxorubicin delivery and efficacy in solid tumors. FASEB J 2011, 25:280-289 